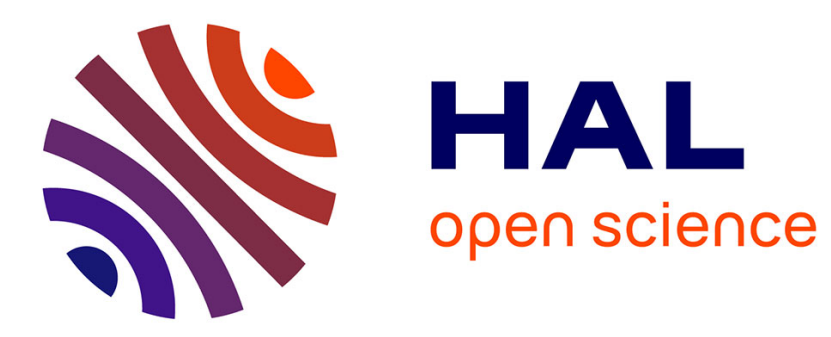

\title{
Les umbos de boucliers de Gournay-sur-Aronde (Val-d'Oise)
}

\author{
André Rapin
}

\section{To cite this version:}

André Rapin. Les umbos de boucliers de Gournay-sur-Aronde (Val-d'Oise). Revue archéologique de Picardie, 1983, Les Celtes dans le nord du bassin parisien. Actes du Ve colloque de l'Association française pour l'étude de l'âge du Fer, 1, pp.174-180. 10.3406/pica.1983.2994 . hal-02536290

\section{HAL Id: hal-02536290 \\ https://hal.science/hal-02536290}

Submitted on 8 Apr 2020

HAL is a multi-disciplinary open access archive for the deposit and dissemination of scientific research documents, whether they are published or not. The documents may come from teaching and research institutions in France or abroad, or from public or private research centers.
L'archive ouverte pluridisciplinaire HAL, est destinée au dépôt et à la diffusion de documents scientifiques de niveau recherche, publiés ou non, émanant des établissements d'enseignement et de recherche français ou étrangers, des laboratoires publics ou privés.

\section{(이) $\$$}

Distributed under a Creative Commons Attribution - NonCommercial - NoDerivatives| 4.0 


\title{
LES UMBOS DE BOUCLIER DE GOURNAY-SUR-ARONDE (Oise)
}

\author{
par André RAPIN *
}

\section{LES DONnÉes de LA fouille}

\section{A. Le contexte du sanctuaire}

La plupart des 2500 objets métalliques trouvés dans le fossé d'enceinte du sanctuaire de Gournay-surAronde sont des armes ou des restes d'équipements militaires, mêlés à un nombre tout aussi impressionnant d'ossements d'animaux ou d'humains sacrifiés.

Une telle quantité d'objets, leur qualité, ainsi que les mutilations diverses qu'ils ont subies avant leur enfouissement, témoignent de l'intense activité religieuse de ce sanctuaire. Depuis le $I l \mid{ }^{\circ}$ siècle avant notre ère jusqu'aux approches de la guerre des Gaules, il a probablement fonctionné sans discontinuer, avec son fossé "d'exposition" ouvert et certainement entretenu, rempli de dépouilles oxydées ou pourrissantes.

Transposée à notre époque, il faudrait imaginer une telle structure ouverte sous Napoléon ler. Les cérémonies et leurs cortèges de sacrifices l'auraient comblée progressivement des dépouilles des grognards de Wagram, puis de celles des combattants des "Trois Glorieuses" et de la Monarchie de Juillet, suivies de l'équipement militaire du Second Empire et de la Troisième République. Sur les niveaux supérieurs, nous rencontrerions les fusils du "poilu" de 14/18, voisinant avec les mitraillettes de la guerre de $39 / 45$, le tout à peine recouvert par les armes plus sophistiquées de nos modernes fantassins.

Bien évidemment, l'évolution technologique fut, sans aucun doute, moins rapide il y a 2000 ans qu'à notre époque. Néanmoins un tel contexte archéologique,

* Institut de Recherches Archéologique et Paléométallurgique, Université de Technologie, 60200 COMPIEGNE ainsi que ces données chronologiques brièvement rappelées, ne sont pas sans conséquence pour la présente étude. Ils font du matériel de Gournay un ensemble tout à fait original, voire unique pour la protohistoire celtique.

C'est d'ailleurs cette particularité qui nous a contraint à innover sans cesse pour pouvoir étudier l'énorme masse d'informations qu'il représente, notamment en créant de toutes pièces un laboratoire de recherche et de restauration.

\section{LES DONNÉES QUANTITATIVES}

\section{A. Données numériques}

$$
\text { relatives au mobilier de Gournay-sur-Aronde }
$$

Près de un dixième du matériel métallique de Gournay, soit 224 objets, a été sommairement identifié et fiché au niveau de la fouille sous l'appellation umbo. Ce chiffre est bien sûr soumis à révision au fur et à mesure de l'avancement des travaux de restauration, et sera remis à jour à la fin de l'étude entreprise.

Après une période de restauration tous azimuts, qui a caractérisé le début de l'activité du laboratoire, nos efforts convergent depuis deux ans sur des catégories d'objets plus limitées. Les umbos constituent actuellement l'objectif prioritaire, et nous envisageons la restauration complète du lot pour 1983 et sa publication pour 1984 (fig. 2). Pour l'instant, près de la moitié de l'ensemble est déjà restaurée (fig. 1), stabilisée, dessinée, étudiée ..., ce qui représente près de 8000 heures de travail

\section{B. Conséquences stratigraphiques}

Le contexte religieux dans lequel baigne tout le mobilier de Gournay-sur-Aronde implique une approche dif- 


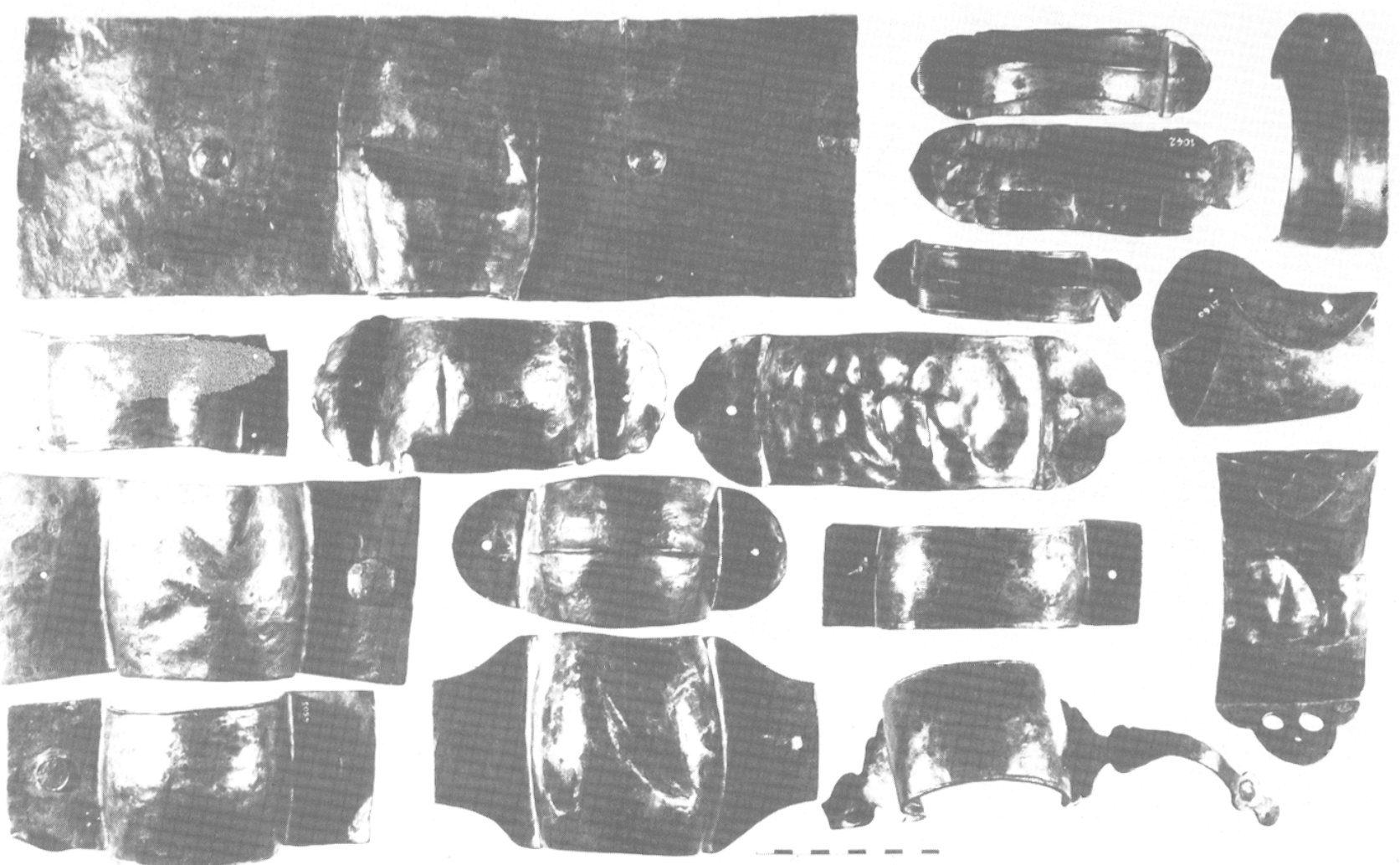

Fg. 1 : Vue d'ensemble d'une partie des umbos apros restauration. 1

Fig. 2 : Etapes classiques de la restauration des umbos.I
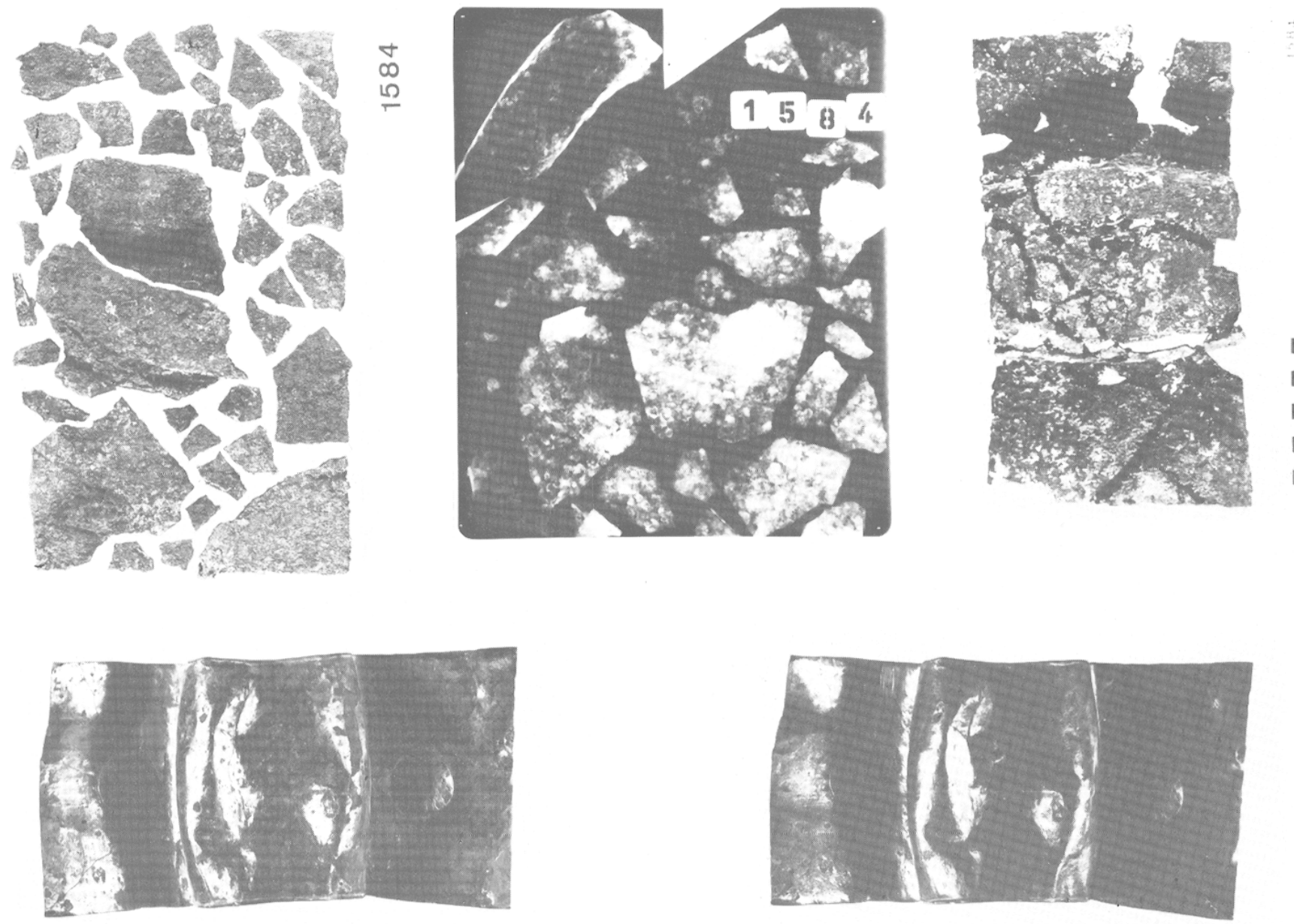
férente de celle relative au mobilier traditionnel des sépultures, ou des habitats. En effet, la longue exposition à l'air des dépouilles (1) ainsi que les manipulations consécutives à "I'entretien" du dépôt, ont pour résultat une stratigraphie très particulière qu'il faut aborder avec prudence.

1. Des umbos, bien que voisins dans l'espace, ne sont pas forcément contemporains, pas plus que leur éloignement en profondeur ne signifie un éloignement chronologique.

2. L'absence de strate dans les terres de remplissage du fossé, probablement dûe à un comblement méthodique et rapide de la structure, ne permet pas les repérages stratigraphiques classiques.

3. Par contre, la "typologie" des sacrifices et le style de certaines mutilations (fig. 3) permettront peutêtre des recoupemients stratigraphiques intéressants avec les différents dépôts mis en évidence par l'étude des ossements (2).

De cet ensemble de constats, il découle que les études morphologiques sommaires, qui sont souvent de règle pour ce type de mobilier lorsqu'il est trouvé dans un contexte de sépulture, sont ici nettement insuffisantes. $D^{\prime}$ où la nécessité de mettre à profit le travail de restauration pour mener à bien une étude morphologique aussi exhaustive que possible de chaque umbo, révélant à la fois la structure interne, la technologie de fabrication, les traces sacrificielles, les décors éventuels e1 la restitution de la forme originelle.

\section{B. Données numériques relatives à l'ensemble du mobilier celtique européen}

Une telle série est, à elle seule, à peu près équivalente à la somme des umbos trouvés sur l'ensemble des territoires de l'Europe celtique. A notre connaissance, les études de synthèse portant jusqu'alors sur ce type de mobilier prennent en compte des séries moins importantes.

1. L'étude du Docteur Jovan TODOVORIC (3) porte sur une série de près de 40 umbos trouvés en Pannonie inférieure et dans la Péninsule des Balkans.

2. Celle de Mieczyslaw DOMARADZKI (4), plus récente, porte sur 143 umbos trouvés sur l'ensemble de l'Europe centrale et orientale. C'est probablement l'importance de cette série, ajoutée à une plus grande rigueur de l'étude, qui explique les décalages chronologiques et typologiques existant entre ces deux études (5).

3. Quant à l'étude de Peter F. STARY (6) sur I'origine et la répartition de l'umbo de bouclier et des boucliers ovales au Second Age du Fer, elle aboutit à des décalages chronologiques encore plus grands et incompréhensibles aussi bien vis-à-vis de notre travail que de celui de $M$. DOMARADZKI, avec lequel nous avons de nombreux points de convergence.

Le matériel de Gournay-sur-Aronde offre en outre un avantage énorme, par rapport à la matière première

Fig. 3 : Typologie des sacrifices de l'umbo (avant ou après destruction du bouclier en bois) et reconstitution hypothétique des instruments du sacrifice.
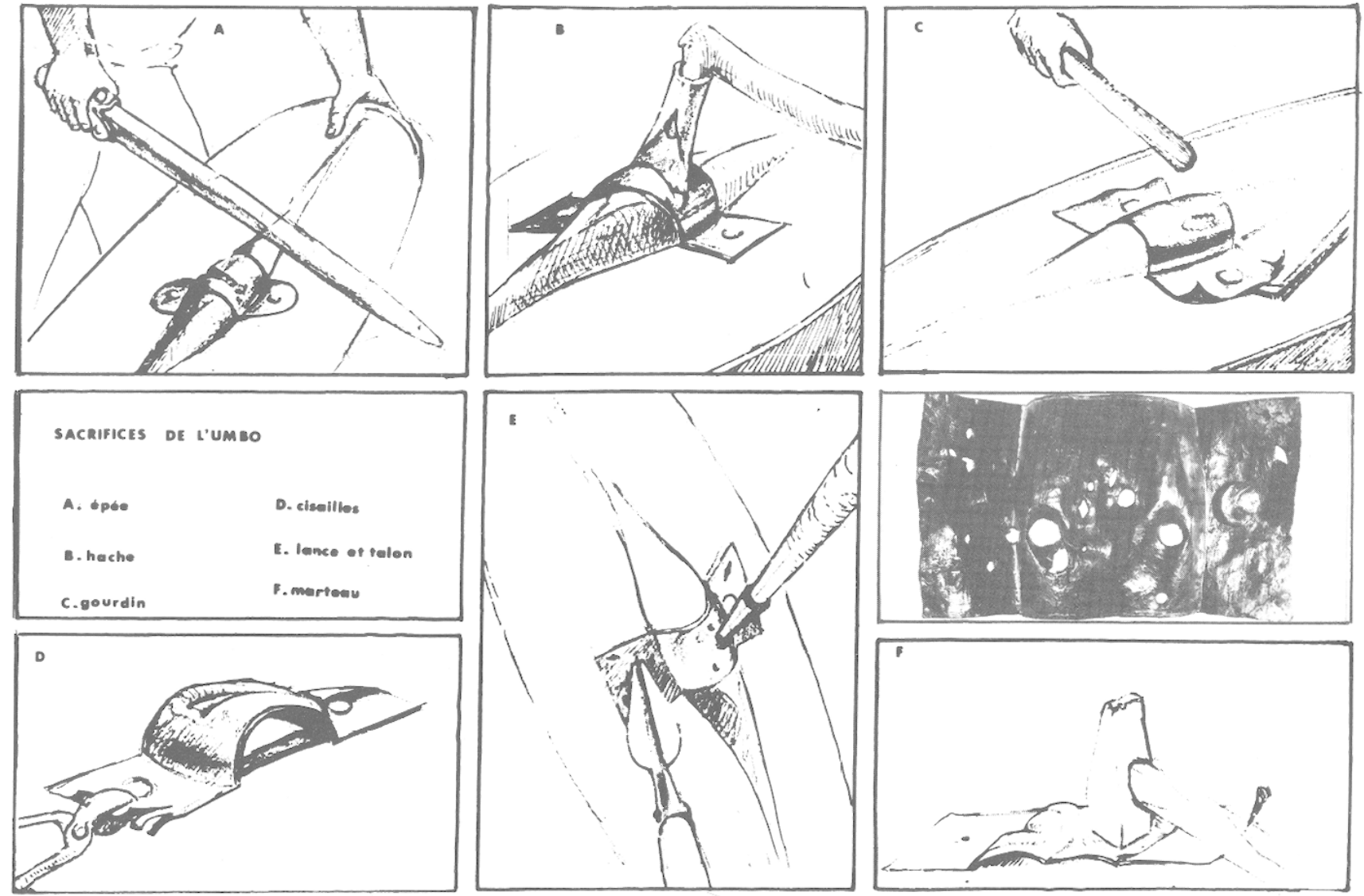
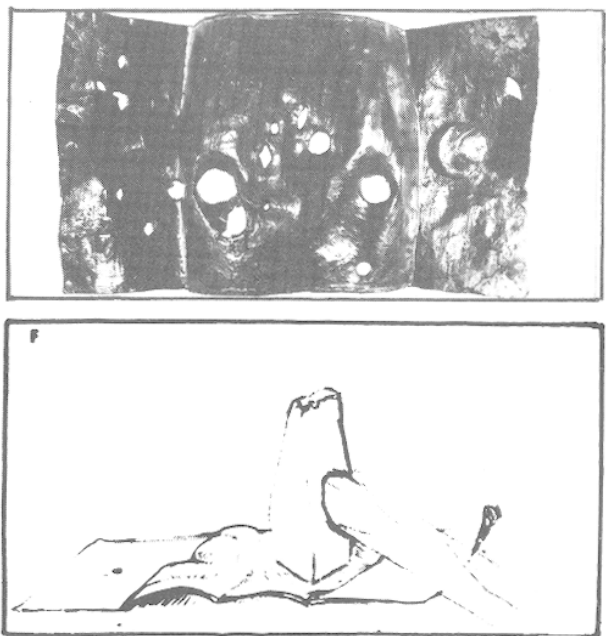
des études citées, et qui va bien au-delà de l'intérêt statistique d'une série plus fiable. II est en effet totalement disponible et sur place, ce qui implique les correctifs constants qu'apportent les informations issues de la fouille et celles provenant des travaux menés simultanément sur le reste du matériel, ainsi que le "feed back" incessant entre l'objet d'une part et d'autre part le restaurateur, le chercheur et le dessinateur parfois confondus en une seule personne.

\section{ETUDE MORPHOLOGIQUE}

\section{A. Vocabulaire et terminologie}

Avant d'aborder l'étude morphologique, nous avons cru bon de préciser le vocabulaire utilisé tant celui-ci est variable d'un pays à l'autre, voire, d'un chercheur à l'autre.

Le mot latin umbo n'est pas universellement adopté et si outre-Manche on lui préfère le terme de "bosse". outre-Rhin on utilise souvent l'expression de "bosse de bouclier en ruban" (Bandschildbückel). De même pour l'umbo composite désigné souvent comme "umbo bipartite", nous avons préféré l'expression umbo bivalve, car, s'il se compose toujours au minimum de deux valves, il est par contre souvent complété par une $3^{\ominus}$ partie (couvre-spina), qui renforce la jointure des deux valves sur la spina (fig. 9)

Les différentes parties de l'umbo classique de Gournaysur-Aronde, à savoir coque, ailes ou ailettes, rivets, bourrelets, nervure médiane ... sont répertoriées dans le schéma des figures 5 et 7 . Quant aux composantes du bouclier lui-même, orles, planche, spina, manipules ... elles sont présentées dans l'éclaté de la figure 6 Cette reconstitution, prenant en compte les matériaux périssables du bouclier, résume les données et les informations recueillies au cours des restaurations d'une centaine d'umbos de Gournay et de quelques autres provenant de nécropoles de Champagne (7).

Fig. 5 : Différentes parties de l'umbo: - 1, coque : - 2, clou ou rivet, -3 cule ou culette ; - 4, spina de bois ; - 5 , manipule de bois ; - 6 , bourrelet correspondant à l'arc de la spina ; - 7, planche. En bas, coupe du montage avec manipule de bois, plus fer:- $a$, épaisseur de la spina sous la coupe ; - b, manipule en bois.

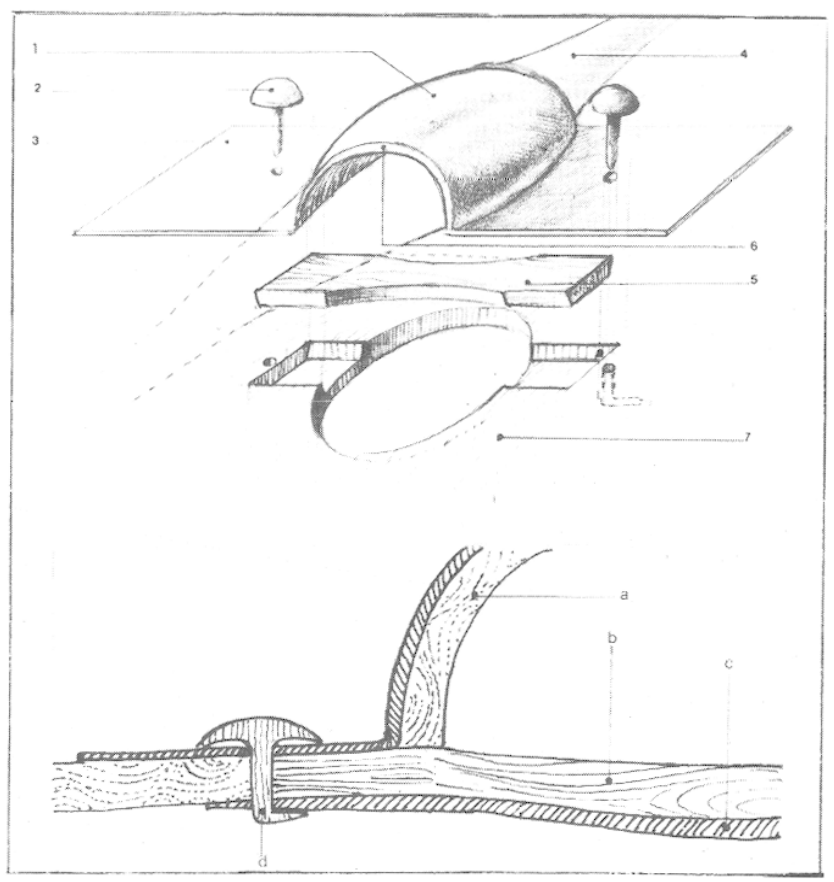

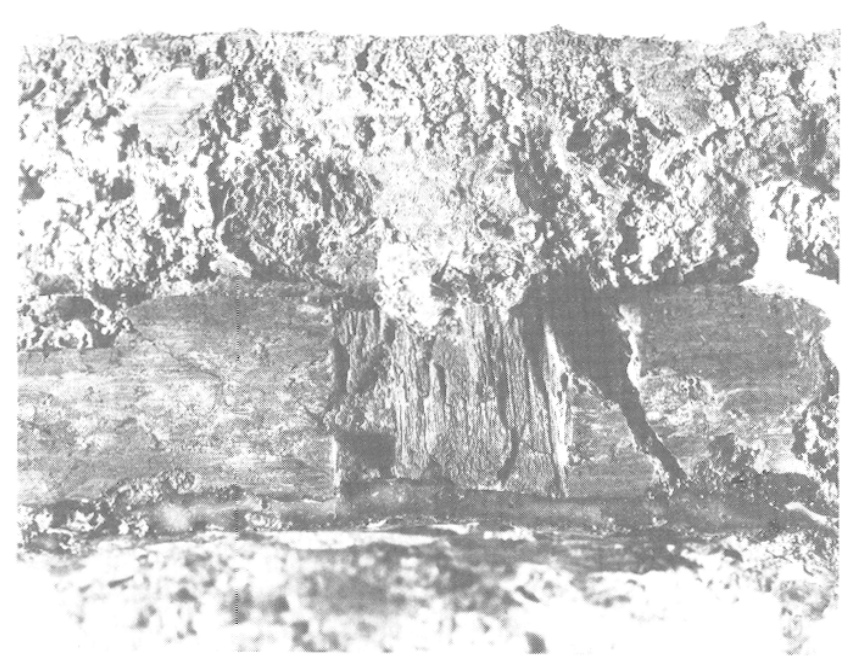

Fig. 4 : Traces de bois sur un umbo du musée d’Epernay (Marne).

\section{B. Mesures}

Pour un tel matériel, déformé par la corrosion, défiguré par les mutilations ou les fragmentations diverses, toute mesure sur un objet non restauré serait sans valeur et pratiquement sans signification (fig. 2). C'est pourtant sur la base de telles mensurations, et donc avec une telle imprécision que sont faites, à ce jour, la plupart des études de mobilier semblable. Cependant, même après restauration, la nature du mobilier de Gournay nous a contraint à nous poser le problème de la pertinence de certaines mesures, telles que la longueur totale de l'umbo ou la hauteur de la coque. Ces mesures sont systématiquement prises en compte, faute de mieux, dans toutes les études un peu précises dont nous avons eu connaissance. Or, il nous a paru absurde de mettre en parallèle des umbos dont la coque, soigneusement aplatie à coups de marteau (fig. 3) augmentait ainsi de longueur, avec d'autres pour lesquels la coque avait été plus ou moins écrasée dans l'autre sens. Ce genre de déformation n'est pas spécifique du site de Gournay; il est également fréquent en sépulture, du fait de l'écrasement dû au poids de la terre de comblement, et il en va de même pour la hauteur de la coque.

\section{Constantes}

La recherche de l'opportunité de telle ou telle mesure nous a également permis de mettre en évidence certaines constantes. Par exemple la largeur de la coque au milieu de l'umbo (liée bien sûr à la largeur moyenne d'un poing fermé) (fig. 8) qui se stabilise aux environs de $100 \mathrm{~mm}(+$ ou $-10 \mathrm{~mm})$, révèle une épaisseur, sous la coque, du bois de la spina de $5 \mathrm{~mm}$ ou $6 \mathrm{~mm}$ au maximum (fig. $5 \mathrm{~B}$ a). L'arc du bord de la coque mesure, pour près de $90 \%$ des umbos, $145 \mathrm{~mm}$ de long $1+$ ou $-5 \mathrm{~mm}$ ) (fig. $5 \mathrm{~A} \mathrm{~b}$ ). L'épaisseur de la planche, révélée par les rivets des ailettes, est de $10 \mathrm{~mm}$ (+ ou $-2 \mathrm{~mm}$ ) (fig. $5 \mathrm{~B} \mathrm{~b}$ ).

\section{Variations}

L'essentiel des variations porte sur :

a) La largeur de l'umbo, qui peut aller de $20 \mathrm{~mm}$ à $150 \mathrm{~mm}$ (ou plus) pour les plus grands (fig. $8 \mathrm{~A}$ et D). 


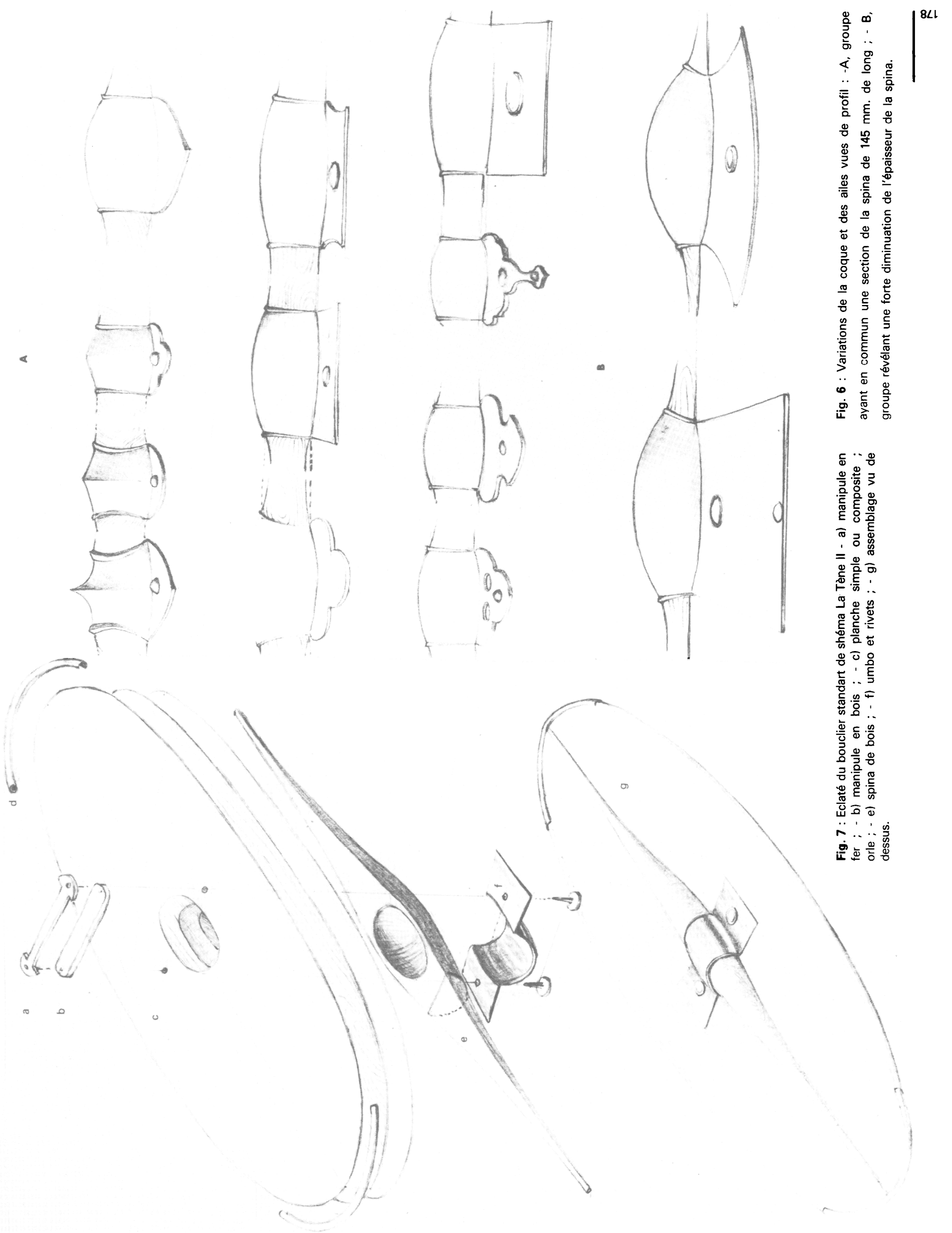




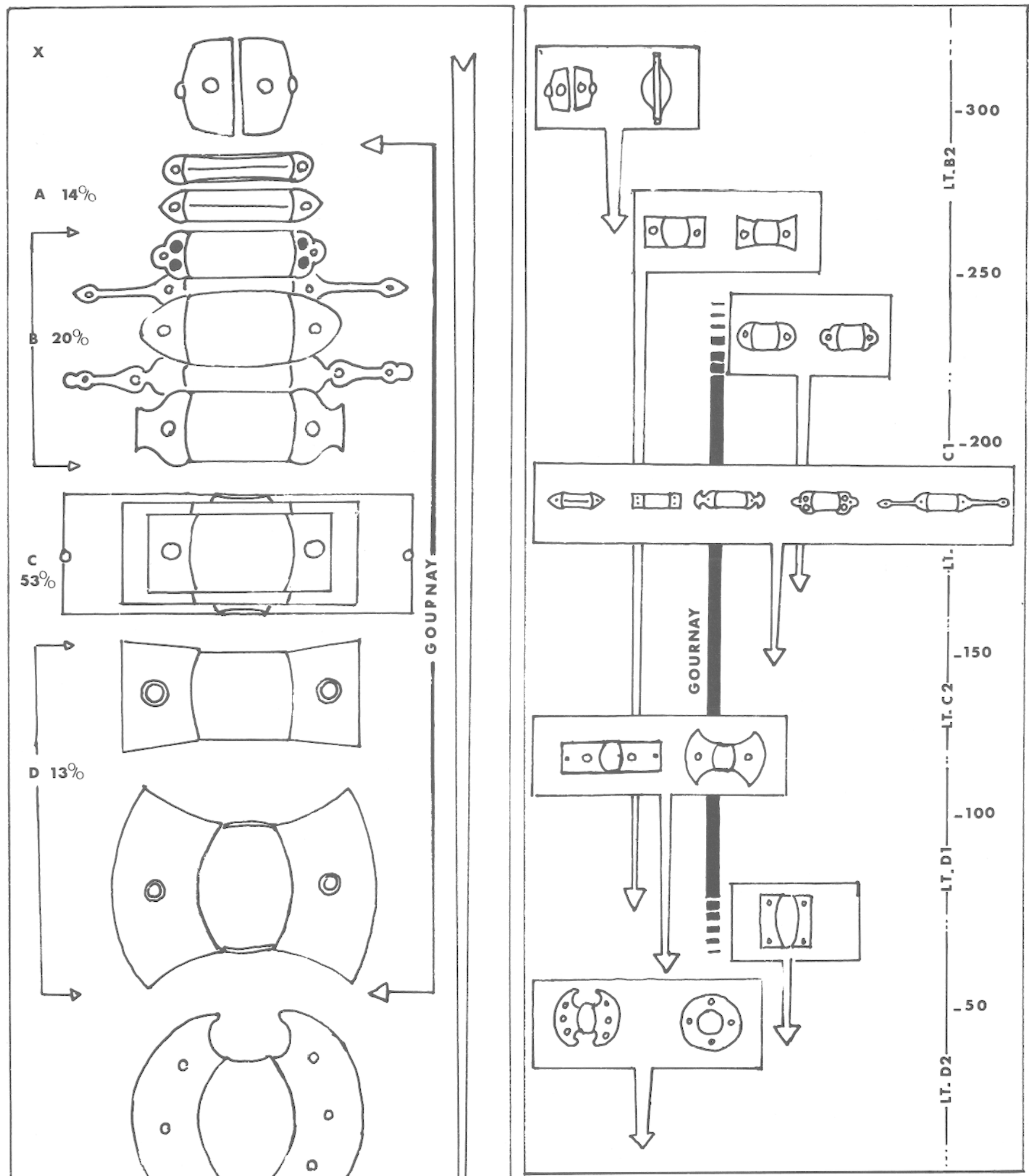

$\Delta$

Fig. 8 : Classement morphologique par taille de l'umbo et formes des ailettes en comparaison avec des types non représentés à Gournay-surAronde ( $X$ et $Y$ ).

Fig. 9 : Première ébauche d'un classement typologique des umbos de Gournay-sur-Aronde et tentative de mise en situation chronologique. 
b) La longueur des ailes, de $20 \mathrm{~mm}$ à $170 \mathrm{~mm}$ (fig. $8 \mathrm{~A}$ et $\mathrm{C}$ ).

c) Leur forme (rectangulaire, ogivale, trilobée, semi-circulaire, etc.) (fig. 8).

d) La forme de la coque, plus ou moins ouverte en demi-tonneau ou demi-cylindre (fig. 8).

e) La présence sur cette coque de certaines moulurations: bourrelets de bordure plus ou moins accentués ou nervure médiane en forme d'arête plus ou moins prononcée (fig. 7)

f) Et enfin les variations relatives aux rivets et affectant leur nombre, forme, dimension ou décor, et la présence associée du manipule en fer.

\section{ClASSEMENT}

Ces différentes observations sont résumées sur le tableau de la figure 8 qui n'est qu'un simple classement morphologique. L'évolution indiquée par la flèche ne doit être confondue ni avec une évolution typologique ni avec une variation chronologique. En effet, certaines formes stables, comme l'umbo à ailettes rectangulaires ou trapézoïdales, se retrouvent d'un bout à l'autre de la stratigraphie du fossé. Cependant il n'est pas rare de déceler au cours de la restauration des variations de structure qui permettront peut-être de préciser une évolution à l'intérieur de ce type, le plus stable et le plus représentatif de cette période (fig. 8 c, $53 \%$ ).

Chaque restauration apporte des informations parfois mineures, mais souvent importantes, voire inédites, comme les rivets enrobés d'une feuille de bronze ou l'apparition d'une nervure inattendue sous la couche d'oxyde. De ce fait, les classements typologiques et chronologiques sont constamment remis en question. Néanmoins, les tableaux ont tendance à se stabiliser au fur et à mesure de l'avancement du travail, celui que nous présentons en conclusion de ce bref aperçu des umbos de Gournay ne sera probablement pas bouleversé de fond en comble d'ici la fin de la restauration de l'ensemble.
Les niveaux les plus anciens du fossé ont livré des umbos à ailettes rectangulaires, parfois très lourds, associés à certains umbos à ailettes semi-circulaires ou trilobées. L'utilisation du fossé comme lieu de dépôt pourrait se situer vers le début de la phase La Tène C 1 (fig. 9). Quant aux couches superficielles, elles ont donné en abondance de très grands umbos, soit aux grandes ailes rectangulaires à deux rivets, soit aux ailes en forme de hache bipenne (fig. $8 \mathrm{D}$ ), qui semblent une préfiguration des formes plus fermées caractéristiques des régions sud-orientales de la Celtique, et même des umbos circulaires présents à Alésia (fig. 8).

\section{NOTES}

1. Voir article de J.-L. BRUNAUX sur les structures et le sanctuaire de Gournay.

2. Voir article de P. MENIEL sur l'étude des ossements animaux du sanctuaire de Gournay.

3. Cf. Dr Jovan TODOROVIC, "Klasifikacya Keltskih Stitova otkrivenik Vesnik", Vojnog Muzeja, 11-12, 1966, pp. 361-374.

4. Cf. Miexzyslaw DOMARADZKI, "Tarcze z okuciami metalowymi na terenie celtyki wschodniej", dans Przeglad Archeologiezny vol. 25, 1977, pp. 53-95.

5. Par exemple, les umbos bivalves n'apparaissent pour J. TODOROVIC, que dans la seconde moitié du $\|=$ siècle, ce qui est totalement incompatible avec toutes les données chronologiques des sépultures contenant ce type d'umbo.

6. Peter F. STARY, “Ursprung und Ausbreitung der einsenzeitlichen Ovalschilde mit spinderförmigen Schildbuckel", Germania, 1981, 59-2, pp. 287-306.

7. Notamment ceux du Musée d'Epernay - Fère champenoise, Faubourg de Connantre - Morains les Terres Rouges - Ecury-leRepos le Crayon - Cette dernière nécropole a fait l'objet d'une publication conjointe avec P. ROUALLET, P. FLUZIN, A. RAPIN et L. URAN dans Mémoire de la Société d'Agriculture, Commerce, Sciences et Arts de la Marne, tome XCVI, 1981, pp. 24-43. 